International Review of Research in Open and Distributed Learning Volume 19, Number 1

February - 2018

\title{
A Taxonomy of Asynchronous Instructional Video Styles
}

Konstantinos Chorianopoulos

Ionian University, Corfu, Greece

\begin{abstract}
Many educational organizations are employing instructional videos in their pedagogy, but there is a limited understanding of the possible video formats. In practice, the presentation format of instructional videos ranges from direct recording of classroom teaching with a stationary camera, or screencasts with voiceover, to highly elaborate video post-production. Previous work evaluated the effectiveness of several production styles, but there has not been any consistent taxonomy, which would have made comparisons and meta-analyses possible. Therefore, we need a taxonomy of instructional video formats that facilitates the understanding of the landscape of available instructional video production styles. For this purpose, we surveyed the research literature and examined contemporary video-based courses, which have been produced by diverse educational organizations and teachers across several academic disciplines. We organized instructional video styles in two dimensions according to the level of human presence and to the type of instructional media. In addition to organizing existing instructional videos in a comprehensive way, the proposed taxonomy offers a design space, which should facilitate choice, as well as the preparation of novel video formats.
\end{abstract}

Keywords: video, instructional, lecture, webcast, podcast, format, production, style, embodiment, instructional media, MOOC 


\section{Introduction}

(M)edia are mere vehicles that deliver instruction but do not influence student achievement any more than the truck that delivers our groceries causes changes in our nutrition. (Clark, 1983, p. 445)

Video lectures have been growing in popularity and many individuals, organizations, and universities are employing them in various instructional frameworks, such as in distance education and in the flipped classroom. Alongside the wide availability of online video lectures on various topics, there is also a wide diversity of video production styles and formats. In many cases, the same topic (e.g., statistics), is transferred on video lecture format with widely different production styles. Thus, every organization, or individual who aspires to create new video lectures, has to make an informed decision among the available video formats, but there is limited documentation on the attributes of each format and the factors that influence this important decision. Although, it costs very little to distribute video to millions of learners, the actual production cost of the video in the first place might be significant. Therefore, there is a need to organize instructional video styles in a simple way that facilitates the choice for the most suitable.

In order to create a taxonomy of instructional video production styles, we need to identify the main sources and representative examples. In addition to the popularity of educational videos on major video streaming sites and repositories (e.g., YouTubeEdu, iTunesU, MIT Open Courseware, VideoLectures.net, TEDEd, etc), there is also a growing number of organizations that offer video-based learning, such as Coursera, Udacity, EdX, Khan Academy, FutureLearn, and Iversity. A survey of the available videos and of the research literature has revealed that the selection of a production styles for a video lecture depends on the instructor's preference and feasibility, or on the organizational (platform) guidelines, rather than a structured theory (Figure 1). For example, all Udacity videos have the same presentation format. On the other hand, the videos on YouTubeEdu (which aggregates material from organizations and individuals) have significant variability. Overall, the most popular instructional video format seems to be the direct recording of the classroom or in the teacher's office with one camera. Nevertheless, what works well in a live classroom might not translate equally well into online video. 

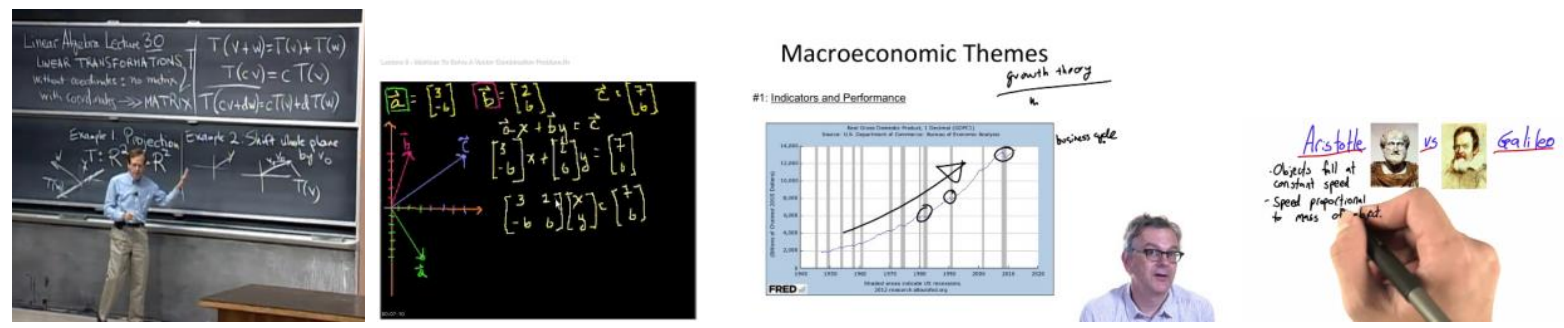

Figure 1. The presentation format of instructional videos ranges from direct recording of classroom teaching with a stationary camera, or writing board screencasts with voice-over, to highly elaborate video post-production with picture-in-picture, or video blending techniques (from left to right: MIT OpenCourseware, Khan Academy, Coursera, Udacity)

There are several video platforms that facilitate established universities and instructors in preparing and sharing instructional video. In addition to the generic video platforms (e.g., YouTubeEdu, iTunesU), in USA there are Coursera, EdX, and Udacity. In Europe the main ones are FutureLearn (UK) and Iversity (EU). Notably, each one of the major video-based education providers seem to feel very confident about its approach to video presentation style and has a consistent style across the video archive and across different subjects and disciplines. For example, Shalman Khan, the founder of the Khan Academy has noted (Thompson, 2011, para. 27): “That way, it doesn't seem like I'm up on a stage lecturing down at you. It's intimate, like we're both sitting at a table and we're working through something together, writing on a piece of paper." On the other hand, the Coursera platform suggests a teacher-centered video format, which presents the teacher either next to the slides, or at an overimposed small window. Finally, Udacity takes the middle road and displays mostly the hand of the teacher, who writes and gestures on an interactive drawing board. Despite the major differences in the production styles, there are also some common patterns, such as the presence of humans and the use of complementary instructional media. In this article, we are exploring the main instructional video classification factors and their nuances.

In the rest of this article, we are going to describe the main factors that define the design of instructional video formats. For this purpose, we have analyzed the state-of-the-art in the research literature and in the industry. The analysis of the research literature was based on extensive Google Scholar keyword searches (e.g., "instructional video" and "video lecture") and the selection of a few recent articles (later than 2010) that have a very good number of citations per year (more than two yearly). The academic articles provided the theoretical groundwork for the taxonomy, which was refined by performing a review of existing instructional video styles in the industry. The review of existing instructional video styles was based on a few major video platforms (YouTubeEdu, iTunesU, Coursera, Udacity, Khan Academy) and aimed at collecting representative examples of different video styles, without regarding their actual popularity or other aspects of quality. 


\section{Academic Research on Instructional Video}

Researchers have recognized that different video production styles might have different learning effects. In the largest study of instructional video formats, Guo, Kim, and Rubin (2014) have identified six basic types of video production style: 1) classroom lecture with instructor on the blackboard, 2) talking head of instructor at desk, 3) digital drawing board (Khan-style), 4) slide presentation, 5) studio without audience, and 6) computer coding session. Some notable findings include that students prefer short videos, slides should include a talking head, the Khan drawing style is more engaging than slides or coding sessions, and the direct classroom recording does not work well online. Nevertheless, in their study, all the courses were from the same platform (EdX) and all the courses are from science and engineering. Although previous empirical research has provided many insights about several video styles, the aggregate results are not comparable because they do not have a common ground with regard to the typology of video production styles. For example, there are many studies that have examined screencasts, but Ilioudi, Giannakos, and Chorianopoulos (2013) and Guo et al. (2014) refer to Khan-style, which is technically a particular type of screencast that records the pen-tip of the presenter on a digital drawing board. Therefore, there is a semantics issue with regard to the unit of analysis that might reduce the understanding, comparison, and extension of previous works.

In order to identify existing instructional video styles and resolve any possible terminology ambiguities, we have organized previous works according to two recurring themes: instructional media (e.g., slides, animation, and type) and human embodiment (e.g., social presence, animated human, talking-head). Indeed, Santos-Espino, Afonso-Suárez, and Guerra-Artal. (2016) examined the instructional video styles in contemporary MOOC platforms and classified them in two main categories: speaker-centric and boardcentric. Although there are few instructional videos that employ just one style, they found that courses on humanities and arts emphasize the former, while science and engineering ones emphasize the latter. Social and life sciences employ a balanced approach between the speaker-centric and board-centric styles. Although they emphasize the use of the terms "speaker-centric" and "board-centric," we could generalize these two terms to the broader notions "human embodiment" and "instructional media" respectively, which are more common in the learning sciences. Moreover, it is worth considering the nuances along the two dimensions: human-embodiment and instructional media.

There are many studies that define a low-level unit of analysis that regards very detailed aspects of instructional media. Sugar, Brown, and Luterbach (2010) have provided an analysis of instructional videos, which are based on the screencasting style (recording of screen). They found that there are two types of screen movement: static or dynamic (follows the cursor). They also found that there are two types of narrative: explicit that describes the exact actions on the screen and implicit, which describes the type of activity on the screen. Swarts (2012) examined screencasting videos with a focus on courses about the use of multimedia software and provided guidelines for the production of good video tutorials that belong to 
the screencasting instructional video format. Video tutorials that explain the use of particular features of computer software are a very popular category and many computer users prefer to watch a demonstration than read a manual. As a matter of fact, the popularity of these video tutorials has also made popular the screencasting style of video instruction. Cross, Bayyapunedi, Cutrell, Agarwal, and Thies (2013) emphasized the use of digital writing on instructional videos and compared the use of handwriting to typefaces. They found that learners preferred handwriting, but they considered the typefaces more legible, so they proposed a middle of the road approach that fades hand-writing into a typeface as soon as a word is complete. ten Hove and van der Meij (2015) analyzed the popularity of instructional videos on YouTube. Although "popularity" is not always correlated with effective pedagogy, it is indicative of contemporary learner expectations. They found that popular instructional videos shared some common characteristics such as fast-pace, text highlights, static images and animations, and high-definition production. Their analysis is the first that correlates particular production elements to effectiveness, but those elements are related mostly to planning and post-production, rather than instructional video format. The above studies provide many insights about the design of instructional media in the context of instructional video, but there is no coherent framework, besides putting them in the same category.

Another major category of studies regard the presence, as well as the type of human embodiment, in the instructional video. Lyons, Reysen, and Pierce (2012) performed a longitudinal study (13 weeks), which compared the use of video lectures with (or without) a video of the instructor at the top left of the screen. They found that students considered the social presence of the instructor in the video to offer more learning. Ilioudi et al. (2013) compared the Khan-style to classroom instructional videos and found that there were no major differences in preference or learning performance between the two conditions. Notably, the comparison between video formats includes a control condition with a printed book. Therefore, video instruction is considered to be a supportive medium and not a substitute for traditional classroom learning. Chen and $\mathrm{Wu}$ (2015) compared three popular instructional video styles: 1) direct classroom recording, 2) studio recorded video lectures with the video of the instructor superimposed to the slides, and 3) office recording of the instructor video next to the slides (voice-over type). Although the latter style includes the presence of the instructor, they refer to it as "voice-over type," because the slides and the voice are the main elements. Kizilcec, Bailenson, and Gomez (2015) compared the constant inclusion of a talking head in the video of a slide presentation to one with moderate presence of the face of the presenter. They found that there were no significant differences in terms of learning performance, but it seems that some students just prefer the presence of the instructor face. Li, Kizilcec, Bailenson, and $\mathrm{Ju}$ (2016) examined the acceptance of a virtual avatar in place of the instructor's talking head. They compare multiple alternatives in the place of the human instructional video, such as animated human, animated robot, and real robot. They found that learners preferred the real or animated human condition to the real or animated robot, but the recall rates were mixed across conditions and genders. Mayer and DaPra (2012) have found that learners prefer a human-like (e.g., voice, eye-contact, and gestures) animated character. In particular, learners preferred real 
human voice rather than computerized voice. The above studies provide many insights about the presence and types of human embodiment in the context of instructional video, but there is no coherent framework, besides putting them in the same category.

Table 1

$\begin{array}{ll}\begin{array}{l}\text { Overview of Keywords Employed to Describe Video Lecture Styles in Previous Related Research } \\ \text { Video lecture style }\end{array} & \text { Reference } \\ \text { screencasting, screen movement, narration } & \text { Sugar et al. (2010) } \\ \text { screencasting } & \text { Swarts (2012) } \\ \text { animated human } & \text { Mayer and DaPra (2012) } \\ \text { social presence } & \text { Lyons et al. (2012) } \\ \text { screencasting, khan-style, handwriting, typeface } & \text { Cross et al. (2013) } \\ \text { khan-style, classroom } & \text { Ilioudi et al. (2013) } \\ \text { khan-style, classroom, studio, office-desk, code, slides } & \text { Guo et al. (2014) } \\ \text { classroom, voice-over, picture-in-picture } & \text { Chen and Wu (2015) } \\ \text { Static pictures, dynamic pictures, text } & \text { ten Hove and van der Meij (2015 } \\ \text { slides, talking head } & \text { Kizilcec et al. (2015) } \\ \text { talking-head, robot, animated human, animated robot } & \text { Li et al. (2016) } \\ \text { speaker-centric, board-centric } & \text { Santos-Espino et al. (2016) }\end{array}$

In summary (Table 1), previous research on the production style of instructional video has provided evaluations of particular video formats, but it has not done so in the context of a consistent taxonomy. The organization of previous research in a table that highlights the main classification factors of instructional video styles facilitates comparisons. Notably, there are some differences between the formats tested by previous research, but the most significant observation is that there are also overlaps and ambiguities due to the lack of a common framework. For example, some works have emphasized low-level elements (e.g., typeface), while other works have employed different terms for similar concepts (e.g., talking head, voiceover). In this way, the production style of instructional videos has been classified in many different, or overlapping categories, which makes it difficult to compare across studies, or to perform meta-analyses. Ideally, a coherent taxonomy would be inclusive of all existing styles and should facilitate the informed 
choice for the production of a new instructional videos. Moreover, a future-proof taxonomy should also hold the predictive attribute for defining new production styles that do not yet exist.

In the next section, we examine contemporary instructional video formats, as found on online learning systems and video archives, in order to identify nuances across the two dimensions of the proposed classification scheme: human embodiment and instructional media.

\section{Taxonomy of Instructional Video Styles}

The availability of instructional video has been increasing since the early 2000's, when broadband access from home became more affordable for more people. Initially, video lectures appeared on generic video streaming sites such as YouTube and iTunes University. Next, video lectures spread quickly to specialized educational organizations, such as MIT Open Courseware, TEDed, and Videolectures.net. Last, but not least, the instructional video format has become even more popular and refined within the Massive Open Online Courses (MOOCs), which have complemented video lectures with other popular e-learning elements, such as syllabus, e-books, assignments, discussion forums, wikis, and peer-grading. Although MOOCs are much more than just video lectures, the MOOC platforms have put a lot of effort in evolving the video lecture format. In this work, we focused on educational videos found on major instructional video repositories (e.g., YouTubeEdu, iTunesU, MIT Open Courseware, VideoLectures.net, TEDEd) and on organizations that offer video-based learning, such as Coursera, Udacity, EdX, Khan Academy, FutureLearn, and Iversity. Besides classification of existing video lecture formats, the proposed taxonomy has put special emphasis on the presentation of the main factors (human-embodiment, instructional media) that define the classification of each video lecture format, as well as on the breadth of existing and possible video formats along the main classification factors. 
Table 2

Index of Instructional Video Styles

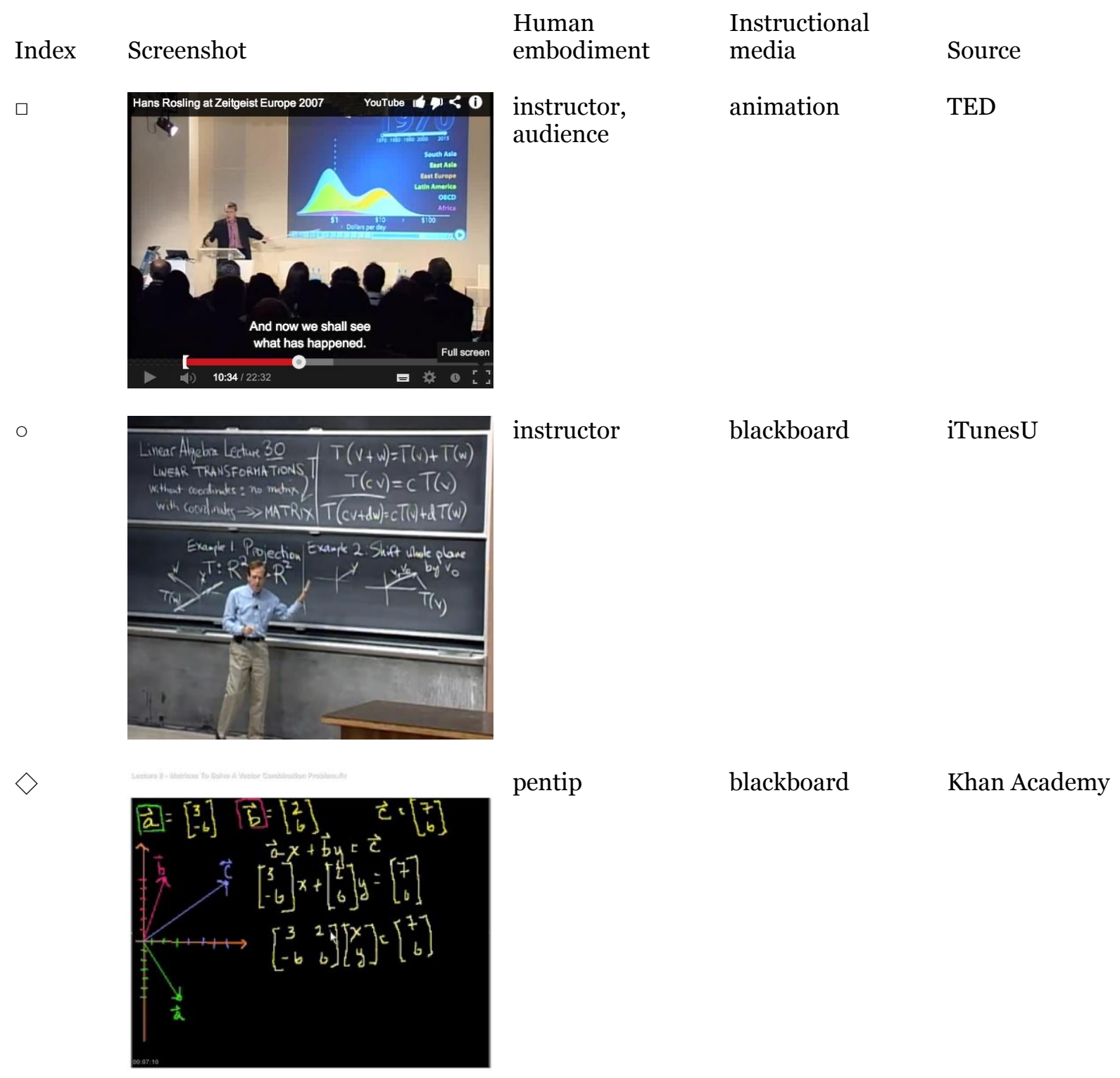




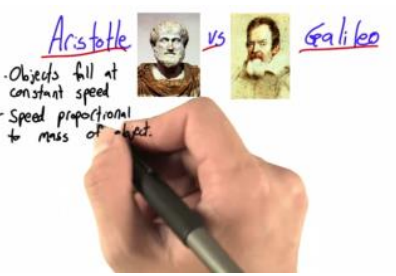

hand

hand

blackboard

Udacity

$\nabla$

Macroeconomic Themes

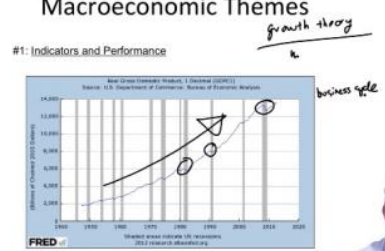

FRED

$\diamond$

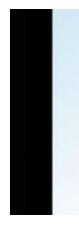

$30-45$ minutes of video lecture | Workload Assigned readings Forum posts/comments

Quizzes
Journal Accomplishment with Distinction)

$\sqrt[3]{3}$
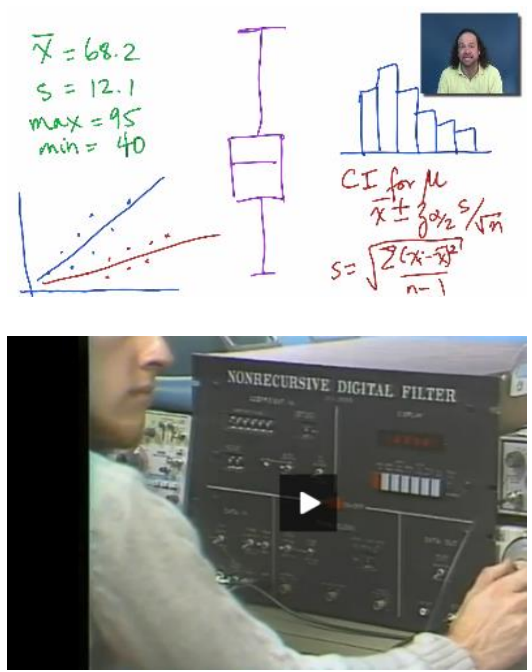

talking-head

slides

pentip

talking-head

talking-head instrument
Coursera

Coursera

\section{Coursera}

MIT

OpenCourseware 
$\triangleleft$
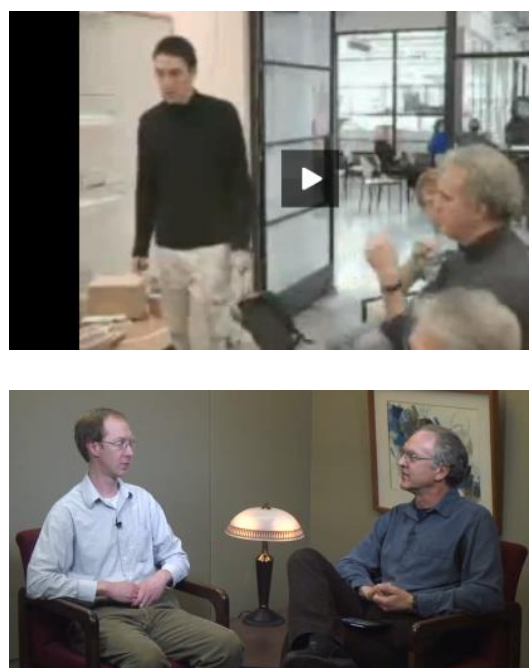

○
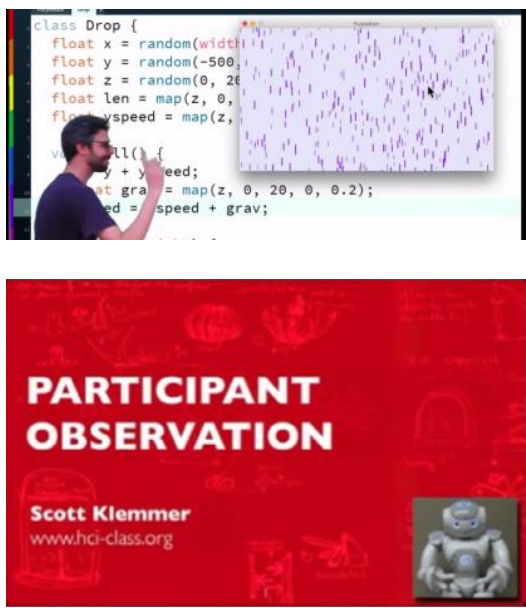

$x$

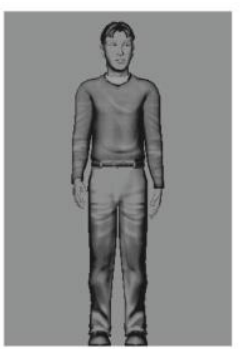

Filled Bonding Sites on outer shell

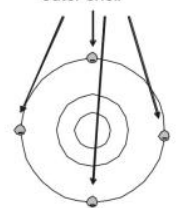

Empty Bonding Sites on outer shell

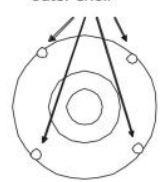

$\odot$

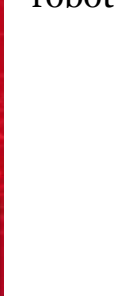

people

instructor

robot

instruments

people

no media

Coursera

live coding

YouTube

slides

animated human slides

Research

sides

\section{Research}

MIT

OpenCourseWare 
We performed a breadth-first random sampling of the available instructional video styles, in order to classify them in Table 2 according to two factors: human embodiment and instructional media. We examined several hundreds of instructional videos, before we could not find any new and significantly different video styles. The theoretical starting point of our taxonomy is the work of Santos-Espino et al. (2016), which we extend by generalizing and by providing a more nuanced spectrum of nominal values along the two dimensions. In the proposed taxonomy, there are two dimension that determine the instructional video style: 1) human embodiment, and 2) instructional media. For each one of the two dimensions there are multiple nominal values that range from the digital (or artificial) to the physical. Firstly, we survey the main instructional video styles in order to assign nominal values to the main attributes, and next, we assign each one on the Cartesian coordinate system with the respective index symbol, which is found on the first column of Table 2 with the nominal coordinates in the next cells of the table, in order to make the final scatter-plot representation more lightweight.

The proposed classification is mostly qualitative and aims to reveal the existing video styles, but it does not provide any information with the regard to popularity, or with regard to learning effectiveness, or suitability to a particular pedagogy, which are left to further work. In particular, the classification factors are nominal rather that quantitative, so the classification is not meant to be absolute about the particular nominal values, rather it is meant to be exact about clearly defining them before measuring them quantitatively. Both classification factors (human-embodiment, instructional media) have the same limits, which are fully digital and fully physical. Thus, the human-embodiment factor has been organized with nominal values that reflect a gamut of human-presence. Similarly, the instructional media factor has been organized with nominal values that reflect a gamut of different instructional media. Then, the mapping of existing instructional video styles on a nominal scatter plot is a straightforward visualization from the classification table. 


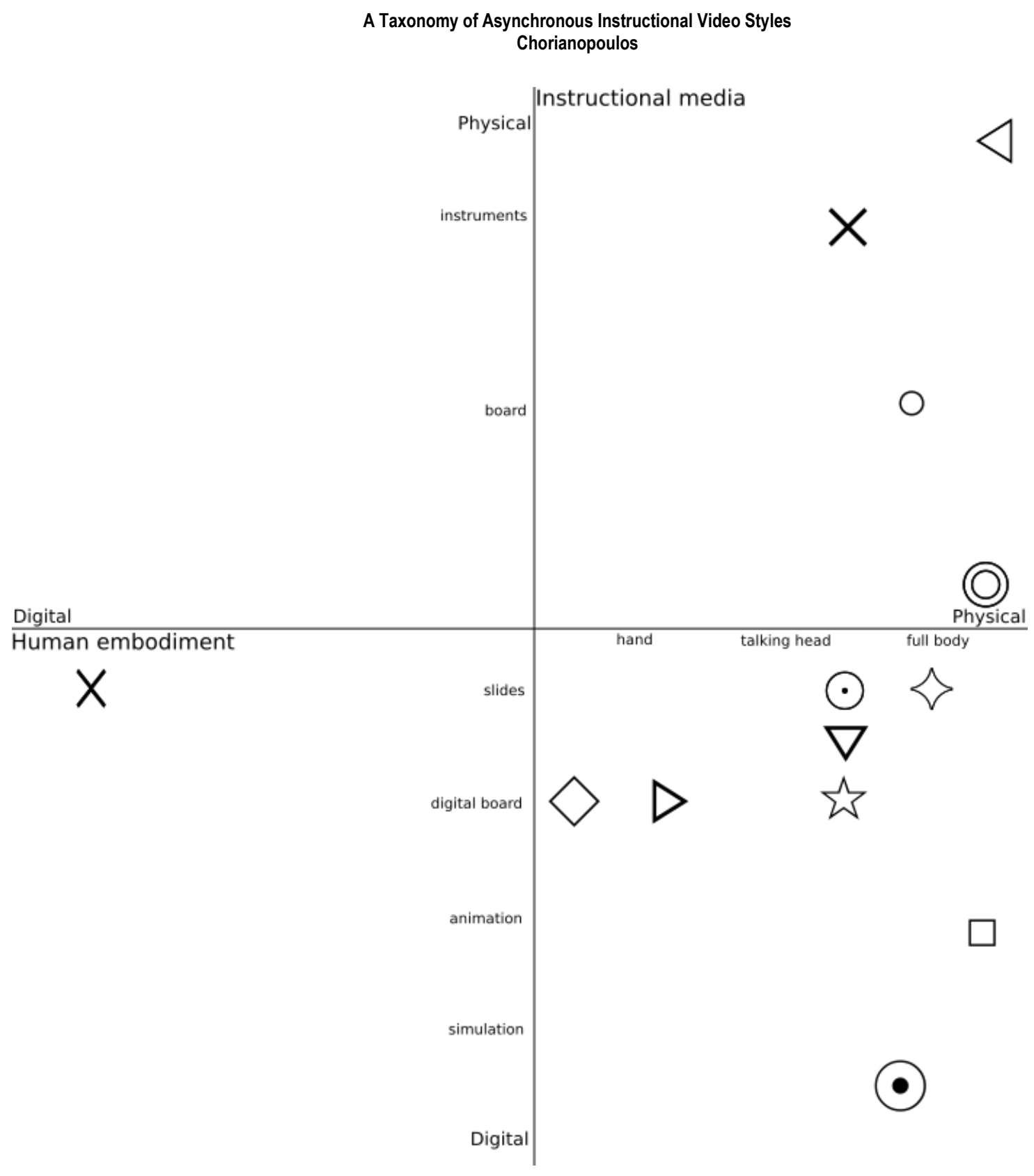

Figure 2. Taxonomy of instructional video styles into two dimensions.

In summary (Figure 2), instructional video formats can be organized into two dimensions: human embodiment and instructional media, which have several nominal values from digital (or artificial) to physical. Although the proposed taxonomy of instructional video styles is just a snapshot of the current situation, the focus of the taxonomy is on the classification factors (human embodiment, instructional media), rather than the details of the production style. Therefore, the discussion that follows is based on 
those factors. The level of human embodiment varies widely between video lecture formats from wide shots that include the audience heads to screen capturing of the tip of the pen. The type of instructional media is another classification factor that varies from slides and animations, to objects manipulated by the instructor. The proposed taxonomy should be useful in understanding the landscape of available options when planning to create a familiar instructional video, or when designing a novel format.

\section{Discussion}

In comparison to previous related work, the proposed taxonomy: 1) holds the predictive attribute, 2) provides a fine-grained spectrum of typologies, and 3) is complemented with a visual representation of the existing and potential video production styles. Most notably, Santos-Espino et al. (2016) have accurately identified two major concepts (speaker-centric, board-centric), but they have only presented them as opposing conditions on one dimension. For example, they characterize existing instructional videos as speaker-centric if the speaker is more important than the instructional media, but, in our view, the speakers and boards are not in a competition for the attention of the learner. In contrast, the proposed taxonomy visually represents speakers and boards as two complementary dimensions and, at the same time, we define a wide continuum of instructional video production styles in a two-dimensional space. Indeed, the survey of existing styles in research and practice has revealed that there is a more fine-grained use of human embodiment and instructional media. For example, speakers might be substituted with digital or artificial agents (animated characters, robots) and boards need not always be digital, but might be physical, too. Finally, the most important contribution of the suggested taxonomy is a comprehensive visual representation of existing and potential new video production styles. In this way, the taxonomy of video formats is more than a map of the current situation; it becomes a tool for navigating toward novel instructional video production styles.

The visual representation of the instructional video taxonomy facilitates a systematic comparison between existing styles, as well as the design of new ones. It demonstrates that we are at an early phase in the development of instructional video styles, because most efforts just replicate the traditional classroom. Notably, there is a vastly underexplored space that regards the employment of artificial representations for humans, such as robots and animated characters. In particular, it is worth exploring the combination of artificial characters with digital media, which might be facilitated by video-game development toolkits. In this way, digital characters might appear to manipulate digital instructional media in the third quadrant. Moreover, there are opportunities in the employment of augmented reality technologies, which bridge physical instructional media with artificial characters. For example, there are TV-studio technologies that enable the tracking of physical objects and enable their interaction with artificial entities (objects or 
characters). In this way, digital (or artificial) characters might appear to manipulate physical objects in the 4th quadrant.

A taxonomy of instructional video would not be complete unless we regarded the broader instructional framework. There are two main approaches to instructional video in education, which define a spectrum of options within them. Instructional video has been used as a substitute for classroom teaching in distance education, or as a complementary instructional tool in flipped-classrooms. For example, a video lecture prepared for distance education of adults assumes that the learner is going to have a minimal contact with the instructor and his peers. On the other hand, a video lecture prepared for K12 students who attend school, assumes that the learner is going to employ the lecture as an instructional medium for home study. Therefore, the motivations and the instructional design of video lectures might be influenced by the target group and the instructional framework. Moreover, according to Anderson and Dron (2010) educational pedagogy could be classified in three generations: cognitive-behaviorist, social constructivist, and connectivist. Contemporary instructional video seems to be at the former stage with videos created by teachers and distributed to learners. It is foreseen that video might be increasingly employed for peer-topeer communication, or remixed and shared between learners and teachers. Therefore, further research could provide a taxonomy of video styles according to the pedagogical approach.
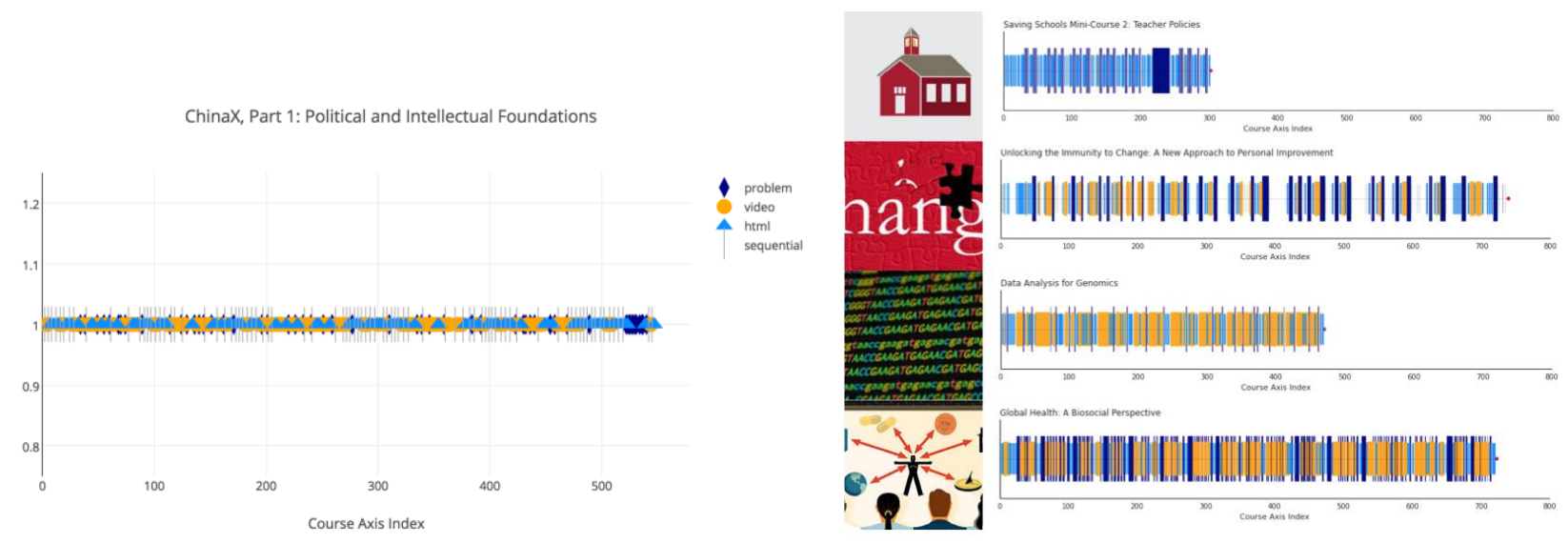

Figure 3. Video is just one component that needs to work with other equally important components (e.g., problem sets, hypertext) (left), while the actual mix of these elements might differ significantly among courses (right).

We did not consider neither the complete instructional design, nor the interaction design aspect of video lectures, but we focused on the visual organization of the video content. Instructional video is a major pillar in pedagogical design, but it is usually complemented with additional types of material (Seaton, 2016), so the selection of a video production style should take a holistic view that considers the type of the course (Figure 3) and the needs of the learners. For example, the Udacity video lectures are much more than video 
recordings of a teacher and instructional media because they are highly structured in terms of learning design and provide the respective user interface that facilitates navigation through video and quiz content. For example, Sebastian Thrun, co-founder of Udacity, has suggested that the video lecture is useful only if it is segmented into many interactive quizzes, which keep the learner engaged just like a video-game does. The same practice is also followed by Coursera, but, currently, the video segmentation seems to be sparser than the one employed by the Udacity system. In addition to video, there are more instructional materials, such as problem sets, hypertext pages, as well as discussion boards. For example, two Stanford professors, Daphne Keller (co-founder of Coursera) and Peter Norvig (instructor at Udacity), have highlighted that their respective MOOC systems are benefited not only by the high quality of the video lectures, but especially by the synchronous assignments, which force students to synchronize and to virtually meet and cooperate in forums and local meet-ups. Thus, further research should evaluate the effectiveness of instructional video styles, in the context of particular pedagogical frameworks.

\section{Conclusion}

In summary, there are some interesting patterns across the evolution and the production style of video lectures. First, video lectures have started as simple recordings of hour-long lectures and have gradually evolved into one minute clips of highly legible and elaborate tablet writing. In particular, there has been an increasing use of technology to manipulate the video recording of the teacher and of the instructional media. Most notably, there is wide variability of human embodiment in the final video, from groups of people, to robots, and digital avatars. In this way, human embodiment and instructional media have been two complementary dimensions in the proposed taxonomy that defines a continuous two dimensional space of existing and potential new instructional video production styles.

Besides the theoretical contribution (disambiguation of terms, visual two dimensional taxonomy), in practice, the proposed taxonomy might facilitate the selection of a video style, or it might even motivate the production of novel ones. For example, a teacher might realize that the screencasting of slides might be enhanced with a drawing board, or a talking head video-feed, which add some extra personality to the final composite video lecture. Moreover, a more ambitious educator with access to studio equipment and skills might realize that there is a vast unexplored space at the third and especially at the fourth quadrant of the visual taxonomy. For example, a possible instructional video format at the fourth quadrant might combine digital avatars that operate on physical instrumentation, which is a common special effects in movies. In this case, we realize that in addition to some serious skills and equipment we also need strong imagination in terms of the possible pedagogies. Although the proposed taxonomy might be a necessary condition, it is certainly not a sufficient one for preparing successful video styles, because effective instructional video is a very complicated topic that also depends on pedagogy and production tools. 
We expect that the experimentation with instructional video styles will continue in a path similar to the experimentation of other linear audiovisual content, such as TV and radio. Indeed, the first TV shows were just radio shows with a static image (Bolter \& Grusin, 2000), but eventually the TV format has evolved in many novel directions. Similarly, we expect that instructional video is going to evolve away from simple classroom recording towards new formats. In the race towards novel instructional video production styles, a few content providers might be able to afford expensive and elaborate styles, which should result in high quality audio-visual appeal and might raise the bar of what is expected by learners. Although the tools of video production have been democratized with inexpensive high-definition cameras and post-processing at the desktop computer, there is still a big difference between an expensive Hollywood-studio movie and a low-budget production. At the same time, we expect that the mainstream instructional video might not be to the liking of everyone. Just like in movies, although production quality is important, there is always an audience for low-budget productions, which have to focus on other aspects, such as originality, narrative, and cinematographic style. 


\section{References}

Anderson, T., \& Dron, J. (2010). Three generations of distance education pedagogy. The International Review of Research in Open and Distributed Learning, 12(3), 80-97.

Bolter, J. D., \& Grusin, R. A. (2000). Remediation: Understanding new media. MIT Press.

Chen, C. M., \& Wu, C. H. (2015). Effects of different video lecture types on sustained attention, emotion, cognitive load, and learning performance. Computers \& Education, 8o, 108-121.

Clark, R. E. (1983). Reconsidering research on learning from media. Review of Educational Research, 53(4), 445-459.

Cross, A., Bayyapunedi, M., Cutrell, E., Agarwal, A., \& Thies, W. (2013, April). TypeRighting: Combining the benefits of handwriting and typeface in online educational videos. In Proceedings of the SIGCHI Conference on Human Factors in Computing Systems (pp. 793-796). ACM.

Guo, P. J., Kim, J., \& Rubin, R. (2014, March). How video production affects student engagement: An empirical study of mooc videos. In Proceedings of the First ACM Conference on Learning@ Scale Conference (pp. 41-50). ACM.

Ilioudi, C., Giannakos, M. N., \& Chorianopoulos, K. (2013). Investigating differences among the commonly used video lecture styles. Workshop on Analytics for Video Learning (Vol. 983, pp. 2126). CEUR Workshop Proceedings.

Kizilcec, R. F., Bailenson, J. N., \& Gomez, C. J. (2015). The instructor's face in video instruction: Evidence from two large-scale field studies. Journal of Educational Psychology, 107(3), 724.

Li, J., Kizilcec, R., Bailenson, J., \& Ju, W. (2016). Social robots and virtual agents as lecturers for video instruction. Computers in Human Behavior, 55, 1222-1230.

Lyons, A., Reysen, S., \& Pierce, L. (2012). Video lecture format, student technological efficacy, and social presence in online courses. Computers in Human Behavior, 28(1), 181-186.

Mayer, R. E., \& DaPra, C. S. (2012). An embodiment effect in computer-based learning with animated pedagogical agents. Journal of Experimental Psychology: Applied, 18(3), 239.

Santos-Espino, J. M., Afonso-Suárez, M. D., \& Guerra-Artal, C. (2016). Speakers and boards: A survey of instructional video styles in MOOCs. Technical Communication, 63(2), 101-115. 
Seaton, D. (2016). Exploring course structure at HarvardX: A New Year's resolution for MOOC research (Blog post). Harvard University. Retrieved from http://vpal.harvard.edu/blog/exploring-coursestructure-harvardx-new-year\%E2\%80\%99s-resolution-mooc-research

Sugar, W., Brown, A., \& Luterbach, K. (2010). Examining the anatomy of a screencast: Uncovering common elements and instructional strategies. International Review of Research in Open and Distance Learning, 11(3).

Swarts, J. (2012). New modes of help: Best practices for instructional video. Technical Communication, 59(3), 195-206.

ten Hove, P., \& van der Meij, H. (2015). Like it or not. What characterizes YouTube's more popular instructional videos? Technical Communication, 62(1), 48-62.

Thompson, C. (2011). How Khan Academy is changing the rules of education (Blog post). Wired. Retrieved from http://www.wired.com/2011/o7/ff khan/

\section{Athabasca \\ University}

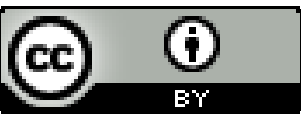

